\title{
Making best systems best for us
}

Penultimate version, forthcoming in Synthese.

\section{Authors:}

Siegfried Jaag: Heinrich-Heine-Universität Düsseldorf

Christian Loew: Université du Luxembourg

\begin{abstract}
:
Humean reductionism about laws of nature appears to leave a central aspect of scientific practice unmotivated: If the world's fundamental structure is exhausted by the actual distribution of non-modal properties and the laws of nature are merely efficient summaries of this distribution, then why does science posit laws that cover a wide range of non-actual circumstances? In this paper, we develop a new version of the Humean best systems account of laws based on the idea that laws need to organize information in a way that maximizes their cognitive usefulness for creature like us. We argue that this account motivates scientific practice because the laws' applicability to non-actual circumstances falls right out of their cognitive usefulness.
\end{abstract}

\section{Acknowledgments:}

Both authors contributed equally to the paper. For helpful comments and suggestions, we would like to thank Chris Dorst, David Glick, Ned Hall, Andreas Hüttemann, Michael T. Hicks, Catherine Jo, Marc Lange, Markus Schrenk, three anonymous referees for this journal, as well as audiences in Cologne and Oxford.

\section{Humean reductionism about laws}

Humean supervenience is

the neo-Humean thesis that every contingent truth about a world-law, dependency hypothesis, or what you will-holds somehow in virtue of that world's total history of manifest matters of particular fact. Same history, same everything. (Lewis 1981: 20)

The "world's total history of manifest matters of particular fact" is usually called the 'Humean mosaic'. It comprises the complete pattern of instantiations of fundamental properties and the spatiotemporal relations between them. ${ }^{1}$ Humean supervenience is Humean because the instantiations of fundamental properties are 'loose' in the sense

\footnotetext{
${ }^{1}$ Lewis proposes that the fundamental properties are perfectly natural and intrinsic (Lewis 1983). This definition of Humean Supervenience might be in tension with fundamental physics, especially quantum physics (see Maudlin 2007). See Lewis (1986a: xi, 1994: 474) for important qualifications. Nothing in what follows hinges on these qualifications.
} 
that they are freely recombinable: no property instantiation has any modal implications for its spatiotemporal neighborhood. ${ }^{2}$

Humean reductionism about laws of nature is the view that the laws reduce to facts about the Humean mosaic. ${ }^{3}$ David Lewis's influential best systems account (BSA), which is based on pioneering work by Mill and Ramsey, provides a two-step recipe for this reduction. First, look for the best systematization of the Humean mosaic. Such a systematization, according to Lewis, is an axiomatized, deductively closed system that contains only truths. Furthermore, the primitive vocabulary in its axioms must refer to perfectly natural properties (Lewis 1983: 367-368). A system is best, just in case it strikes an optimal balance between strength and simplicity. Strength measures the information content of the system and simplicity the (syntactic) complexity of its axioms. ${ }^{4}$ Second, the laws are identified with the contingent regularities that are axioms or theorems in the best system (Lewis 1994: 478). Lewis (1983: 367) initially suggests that if different systems are tied for best, a contingent regularity must be part of all best systems in order to be a law. In later works (1994: 479), he argues that in such circumstances there would not be any laws, though he maintains that such ties are unlikely.

The guiding idea behind the BSA is that the physical laws are not identified in isolation as in the case of traditional regularity-theories (see Hempel 1965). Instead the laws are those regularities that can be integrated into a system that as a whole describes as much of the world as possible in as compact a way as possible (see Lewis 1983: 367). The BSA promises to distinguish laws from non-laws without positing any metaphysical structure over and above the Humean mosaic. Moreover, it arguably draws this distinction by appealing to the same theoretical virtues that physics itself uses when discovering laws (see Lewis 1983: 367 and 1986a: 123; cf. Earman 1986: 88 and Loewer 2007: 320). Much recent discussion of the BSA has focused on how the

\footnotetext{
2 Some amendments of free combination are necessary to deal with fundamental quantitative properties or (vectorial) magnitudes (see Dorr and Hawthorne 2013, Hall 2012, Hawthorne 2006, and Weatherson 2015). Free recombination enables a reductive account of modality (see Lewis 1986b; cf. Wilson 2015).

${ }^{3}$ See Lewis (1988: 30-31, fn. 15). If we talk about laws (of nature) in the following, we mean the fundamental laws of physics, as opposed to the so-called laws of the special sciences. ${ }^{4}$ See Lewis (1973: 73). Lewis (1994) introduces the further condition of 'fit' to cover chancy laws. Roughly put, a system that assigns the actual history of events a higher chance of happening has a better fit than one that assigns it a lower chance. For our purposes, however, addressing non-chancy laws is sufficient.
} 
notions of strength and simplicity ought to be understood and whether the BSA really succeeds in correctly delineating the laws from the non-laws (see, e.g., Earman 1984, 1986, Loewer 1996, Roberts 2008, and Woodward 2014).

In this paper, we will use a general challenge for Humean accounts of laws to motivate a new version of the BSA. Even if a Humean account of laws correctly delineates the laws from the non-laws, it still needs to explain why, in light of a Humean metaphysics, it makes senses that physics distinguishes between laws and non-laws in the way it does. In particular, it is puzzling from a Humean perspective why physics seeks laws that have modal latitude, that is, cover a wide range of non-actual circumstances (see Hall 2012, 2015). Our goal is to develop a version of the BSA that meets this challenge. ${ }^{5}$

The paper has the following structure: First, we argue that orthodox versions of the BSA leave unexplained why science aims for laws that display modal latitude (§2). Second, we defend a reformed version of the BSA according to which laws are calibrated toward providing information that is cognitively useful for limited creatures like us (§3). And third, we argue that our version of the BSA explains the modal latitude of laws that is implicit in scientific practice $(\S 4)$. We conclude by comparing our account to other recent versions of the BSA that also emphasize the importance of our cognitive limitations $(\S 5)$.

\section{The challenge from scientific practice}

Physics arguably aims to discover the fundamental laws of nature and distinguishes them from accidental facts that can, in principle, be explained in terms of the laws. A philosophical theory of laws should explain why, in light of its metaphysics, the laws have this relevance for science. Why, in Hall's (2015: 268) words, are the laws "distinctively appropriate targets for scientific inquiry?"

This latter requirement poses a special challenge for Humeans. Non-Humeans hold that the laws are grounded in a distinctive kind of fundamental modal structure. ${ }^{6}$

\footnotetext{
${ }^{5}$ Cohen and Callender (2009) and Hicks (forthcoming) defend Humean accounts of laws that are (partly) similar in spirit to the one we will propose. We contrast our account with their respective accounts in section 5 .

${ }^{6}$ For instance, Armstrong (1983) identifies laws with instantiations of an irreducible higherorder necessitation relation between first order universals. Maudlin (2007) regards the fundamental (dynamical) laws as sui generis entities. Bird (2007) argues that the laws originate
} 
They can then appeal to this extra structure to explain why the laws are of special interest to science. Humeans, by contrast, hold that the world fundamentally just is an arrangement of non-modal properties. So, there is no distinctive metaphysical structure that demarcates laws from non-laws. Why then are some facts about the arrangement of non-modal properties (viz., facts that have the status of laws) of special interest to science but not others (viz., facts that do not have the status of laws)?

Hall $(2012,2015)$ has forcefully argued that existing Humean theories of laws cannot fully explain the laws' importance for science. If all that exists is the Humean mosaic, it would be natural for science to aim for laws that encode as much information as possible about the mosaic in as simple a form as possible. This idea is echoed in Lewis's BSA. Lewis (1983: 367) says the following about the virtues of best systems:

I take a suitable system to be one that has the virtues we aspire to in our own theory-building, and that has them to the greatest extent possible given the way the world is. It must be entirely true; it must be closed under strict implication; it must be as simple in axiomatisation as it can be without sacrificing too much information content; and it must have as much information content as it can have without sacrificing too much simplicity.

So the members of Lewis's best system jointly make up the most efficient summary of the Humean mosaic. From the perspective of a Humean metaphysics, it would then appear to make perfect sense to treat all of these facts as laws. But, in fact, science does not regard some of these facts as even serious candidates for laws. (Note that Lewis's BSA arguably already takes this fact into account by reserving law status for just the regularities in the best system. We will come back to this point shortly.)

Hall (2015: 270) points out that if science aimed at discovering the most informative and simplest systematization of the Humean mosaic, then what he calls the "phony fundamental constant" would be a serious candidate for a law. The phony constant is a single real number that encodes the entire physical state of the universe at an instant: "just take all the coordinates, masses, and charges of all the particles, expressed in decimal notation, and interleave the digits" (ibid.). A system that combines the phony constant with two-way deterministic dynamical laws encodes every truth 
about the universe in a very simple form. The phony constant specifies the complete state of the universe at one time, the laws then determine its state at all other times.

The phony constant, however, is clearly not a law of nature. Physicists might be very interested in knowing the total state of the universe at a time that the constant encodes. But they would still treat it as a mere accidental fact that can, in principle, be explained in terms of the (dynamical) laws and initial conditions (see Maudlin: 174175). Moreover, the laws of nature determine nomological possibility such that circumstances are only nomologically possible if they are compatible with the laws. So, if the phony constant is added as a law to two-way deterministic dynamical equations, then, as Hall (2015: 269) points out, it "will shrink the set of nomological possibilities down to one. But that is a disaster, for [...] it is a non-negotiable desideratum on an account of laws that it yield a non-trivial distinction between what is nomologically possible and what is not." Hence, the phony constant shows that facts that one might expect to be laws given a Humean metaphysics are not regarded as candidates for laws by physicists.

Let us add two clarifications about the phony constant. First, it might be objected that because of its huge number of digits the phony constant is not syntactically simple and so will not be part of the simplest, most informative systematization of the Humean mosaic. However, as Hall (2015: 270) points out, our actual laws contain constants that are syntactically just as complex as the phony constant. So, any standard of simplicity that would rule out the phony constant would also rule out these legitimate constants. Second, the phony constant also is not ruled out by Lewis's (1983: 367-368) requirement that the primitive vocabulary in the best system only refers to natural properties. Since the phony constant concerns only particle positions, charges, and masses, which are prime candidates for being perfectly natural properties, it is compatible with this naturalness-constraint (cf. Hicks forthcoming).

At this point, Humeans may concede that facts like the phony constant can be part of the best system yet deny that these facts thereby count as laws. In fact, you might think that this was the idea all along. Here is Lewis:

The ideal system need not consist entirely of regularities; particular facts may gain entry if they contribute enough to collective simplicity and strength. (For instance, certain particular facts about the Big Bang might be strong candidates.) 
By restricting the laws to regularities, Lewis allows that particular facts, such as the phony constant, may enter the best system yet still insists that they are not laws.

But that particular facts such as the phony constant may be part of the best system still is troubling for Humean reductionism. First, it is not clear that Lewis's restriction that only regularities can be laws is scientifically motivated. As Maudlin (2007: 11-12) points out, it is highly unnatural to think of, for example, Newton's second law or the Schrödinger equation as regularities. Moreover, some alleged candidates for physical laws are clearly not regularities. For example, Feynman (1965: 116) proposed that it is a law that the universe started in an initial state of low-entropy (see also Albert 2000, 2015). So, an adequate account of laws should not rule out that some non-regularities are laws a priori (see our discussion in section 4 below).

Second, an adequate theory of laws not only needs to get their extension right but also needs to explain their importance for scientific practice (see our discussion of Loewer's (2007) account below). So, even if it were true that all laws are regularities, it is still not clear how this 'formal' feature would explain their importance to scientific practice. After all, non-regularities in the best systems would be just as much part of the world's best systematization as regularities. So, why does the mere fact that they are not regularities disqualify them from having the same relevance for science as laws? At the very least, Lewis would need to explain what is so special about regularities.

And, third, an analogous challenge as the one posed by the phony constant arises with respect to regularities. Suppose it is true that the "total quantity of electric charge at each moment is Q" (Lange 2009: 57). This regularity is a natural candidate for being part of the best system. As seen above, Lewis admits particular facts (for instance about the Big Bang) in best systems. Indeed, he (1986a: 123) explicitly allows that the laws themselves may refer to particular facts. So, the best system may contain the fact that the total charge of the universe at some particular time is Q. And suppose also, as is plausible, that it is a true regularity in the best system that charge is conserved. These two facts together then entail the above regularity. Moreover, since best systems are deductively closed (Lewis 1994: 478), this regularity will itself be part of the best system. Alternatively, this and similar regularities may enter the best system 'directly'. They would add an enormous amount of information while making the system only 
slightly less simple (Lange 2009: 57 and Woodward 2014: 97). So, these regularities would be perfect Humean candidates for laws. Yet, actual physics does not treat regularities about the total charge of the universe (or other specific quantities) as serious candidates for laws of nature. So, restricting the laws to regularities still leaves unmotivated why science draws the distinction between laws and non-laws in the way it does.

Moreover, there is a second aspect to Hall's challenge that is equally troubling for Humean reductionism. The facts that science does regard as serious candidates for laws do not appear to be natural candidates for laws in light of a Humean metaphysics. Following Hall, it will be helpful to divide the information content of the fundamental physical laws into two factors:

[T] he fundamental laws "factor" the set of nomologically possible total histories of particle behavior into an initial conditions hypothesis (ICH) and a dynamical hypothesis (DH). (Hall 2015: 263)

The ICH delimits "a set of nomologically possible initial conditions" (ibid.). The DH specifies "how each such initial condition would evolve forward in time" (ibid.). In other words, the information content of a set of laws gets factored into: (i) what initial conditions they allow; and (ii) how they restrict the evolution of the universe as a whole given each such condition.

Hall points out that theories that fundamental physics takes seriously as candidates for the fundamental laws have a strong DH but a weak ICH. It is easy to motivate that physics aims for a strong DH. The gold standard for a strong DH is determinism, which allows given the world's initial state only one way for it to evolve (cf. Earman 1984, 1986). Many candidates for fundamental laws from the history of physics including Newton's laws, the laws of (classical) electrodynamics, and the laws of relativity are deterministic. And even when determinism is in doubt, such as in quantum mechanics, scientists express a clear preference for determinism. For instance, Einstein has famously insisted that "God does not play dice."

It is equally plausible that science aims for laws that have a weak ICH. Hall points out that Newton's laws are preferable over Kepler's laws of planetary motion because the former answer "questions not merely about how the elements of the solar system did, do, and will behave, but also about how they would have behaved under alternative 
physical conditions" (Hall 2015: 263). In general, most candidates for physical laws provide lots of information about what would have happened if the initial conditions were different. For example, Coulomb's law determines the electrostatic forces between charges $q_{1}$ and $q_{2}$ at a distance $r$ for any values of $q_{1}, q_{2}$, and $r$. Taken at face value, Coulomb's law ranges over all values of these variables, not just values that are actually instantiated. So, it applies to a wide range of merely possible initial conditions.

It is not part of the proposal that laws apply to every metaphysically possible initial condition. For example, every set of laws arguably rules out initial conditions that include 'alien properties', i.e., properties that are not instantiated in the actual world (see Hall 2012: 47-49). Moreover, several non-dynamical laws rule out metaphysically possible initial conditions. For instance, the Wiedemann-Franz-law entails that every thermally conductive piece of metal is also electrically conductive. So, this law excludes metaphysically possible initial conditions where, for instance, a piece of metal is thermally conductive but not electrically conductive (or vice versa). Other possible candidates for fundamental physical laws place even stronger restrictions on the initial conditions. As mentioned above, it may be a fundamental physical law that the universe started in an initial state of low-entropy (see Albert 2000). Adding this restriction to a set of laws would rule out all initial conditions where the universe does not start in an initial state of low-entropy. But even such laws still would have a comparatively weak ICH in that they allow a significant range of possible initial conditions. For example, a law that dictates that the universe starts in a low-entropy state still allows different ways for this condition to be microscopically realized. These differences in microstates, combined with plausible dynamical laws, then allow for things being very different from how they actually are, including circumstances where no galaxies form or no life exists in the universe.

Hall argues that Humean reductionism has no ready explanation for why science aims for laws that have a weak ICH. A set of laws that has a weak ICH applies to a wide range of other possible initial conditions. We will call this feature modal latitude:

Modal latitude. A set of laws has modal latitude, just in case it permits a wide range of possible initial conditions. 
Modal latitude allows a set of laws to answer "questions both about what would have happened, and about what would have to have happened, had conditions in the world been different in some specified respect." (Hall 2015: 271; italics in the original). This aspect of the laws seems to make sense for non-Humeans, which hold that the world has fundamental modal structure. The modal latitude of the laws of nature then arises because they map out this modal structure. ${ }^{7}$ But what is the purpose of modal latitude for Humeans? If Humeanism is true, then the world has no modal structure that is metaphysically prior to the laws of nature. So, there is no antecedent modal structure for the laws to describe. Why then is science interested in laws that apply to a whole range of merely possible initial conditions? Allowing non-actual initial conditions, after all, comes at the cost of providing less information about what actually happens.

We now have the pieces in place to formulate Hall's challenge for Humean accounts of laws. Facts that one would expect to be prime candidates for laws given a Humean metaphysics (because they encode a lot of information about what actually happens, such as the phony constant) are not considered as such by fundamental physics. At the same time, the facts that fundamental physics does take seriously as laws seem to be poor candidates for laws from a Humean perspective (because they talk about other possible initial conditions at the cost of providing information about the actual mosaic). So, the standards of lawhood that physics in fact employs appear to be out of touch with a Humean metaphysics. Humeans need to meet this challenge by clarifying how they can motivate the standards for lawhood that physics in fact employs. Else, there is the risk that their account has revisionary consequences for scientific practice that arguably do not arise for non-Humean accounts.

Meeting this challenge requires more than spelling out an account that correctly predicts what the actual laws are (see Hall 2015: 272). As Lange's (2009) and Woodward's (2014) criticisms indicate, Lewis's BSA may fail to correctly delineate the laws from the non-laws. Proponents of the BSA, however, may simply tweak Lewis's criteria for lawhood to ensure it gets the distinction right. Hall (2015: 266) calls the "unofficial guiding idea" of Lewis's BSA the claim "that our implicit scientific standards

\footnotetext{
${ }^{7}$ For example, Lange's (2009) non-Humean account according to which, roughly put, what makes something a law is that it is invariant under a wide range of counterfactual perturbations seems to be tailor-made to capture the modal latitude of laws. We are skeptical, however, that non-Humeans accounts, in general, offer satisfying explanations of the modal features of laws (cf. Jaag 2014).
} 
for judging lawhood are in fact constitutive of lawhood." The official idea is that these standards are strength and simplicity. But you might interpret these virtues as mere stand-ins for whatever standards sciences in fact uses when discovering laws. A system then is best, just in case its members jointly best satisfy these criteria (cf. Loewer's 2007: 324). This version of the BSA then gets the distinction between laws and nonlaws right by fiat. ${ }^{8}$ For example, since obviously, the standards actual physics uses rule out the phony constant as a law, if we use the very same standards as criteria for the bestness of a system, the phony constant will also not be part of the best system.

But such an account does nothing to explain why the criteria that science in fact uses to identify laws are motivated by Humean lights. The above discussion shows that the standards of lawhood implicit in scientific practice favor laws that have modal latitude. Why, given a Humean metaphysics, does it make sense for science to aim at discovering laws with this feature? To answer this question, Humeans have to motivate the standards for lawhood implicit in scientific practice.

In the rest of the paper, we develop and defend a way of motivating these standards: making best systems best for us. We will argue that the laws' modal latitude is a by-product of their cognitive usefulness for creatures like us. We will first show that it is an independently plausible Humean constraint on laws that they need to be useful to limited beings like us $(\S 3)$. We then show that the modal latitude of the laws of nature falls right out of this constraint $(§ 4)$.

\section{Best systems that are best for us}

In this section, we will argue that best systems need to provide information that is useful for limited beings like us. Not all truths about the Humean mosaic are equally useful for limited beings. Imagine a world almanac that lists every particular truth, one after another. This information, though abundant, would be very hard to store and it would be difficult to extract relevant bits from it. Truths that are useful for limited

\footnotetext{
8 There is one outstanding worry for this proposal. The epistemic standards that science uses to discover laws may be tied. That is, two or more systems may satisfy these criteria equally well. If Humeans then take these epistemic standards as constitutive of laws, it would, in this hypothetical case, be metaphysically indeterminate what the laws of nature are. But this consequence is implausible. This worry seems to be at the heart of the two hypothetical scenarios that Hall (2015: ch. 17.7) discusses as further challenges for Humean reductionism in the conclusion of his article. Discussing this challenge is a topic for another paper.
} 
beings need to be organized more efficiently even if that comes at the cost of losing information.

Philosophers frequently use the idea that information about the Humean mosaic needs to be organized around the cognitive needs of limited beings like us to motivate the BSA. David Albert imagines an audience with God where you get to ask about the world. Albert points out that God listing every single truth about the world would be of very little use to limited creatures like us:

[Y] ou explain to God that you're actually a bit pressed for time, that this is not all you have to do today, that you are not going to be in a position to hear out the whole story. And you ask if maybe there's something meaty and pithy and helpful and informative and short that He might be able to tell you about the world which (you understand) would not amount to everything, or nearly everything, but would nonetheless still somehow amount to a lot. Something that will serve you well, or reasonably well, or as well as possible, in making your way about in the world. (Albert 2015: 23)

Albert emphasizes that beings like us have limited resources: you are "a bit pressed for time" and not in "a position to hear out the whole story." You are better off with something that is cognitively more tractable, even if that means losing some information content, as long as the result is still informative enough for "making your way about in the world." Beebee (2000: 547) similarly points out that the information in best systems needs to be "comprehensible to our feeble, finite minds." Despite its prominence in informally glossing the BSA, the idea of fitting the laws to the cognitive needs of limited beings has played very little role in systematically developing the view. ${ }^{9}$

For Humeans, the laws do not latch on to any pre-existing modal structure that delineates facts that hold as a matter of law from merely accidental facts. It is then extremely natural to think that what distinguishes the laws has, at least in part, to do with us. As Hall (2015: 268) puts it, "[h]ow could the details of our peculiar human situation not be relevant to this matter?" In what follows, we will take up this suggestion and defend a version of the BSA that explains the laws' distinctive status for science in terms of their usefulness for limited creatures likes us. We will argue that this version of the BSA meets the challenge for Humean accounts we have outlined in the previous

\footnotetext{
${ }^{9}$ Hicks's (forthcoming) "Epistemic Role Account" and Cohen and Callender's (2009, 2010) better best system account are an exception. We compare them to our account in section 4 . Dorst (forthcoming) develops a similar view to the one discussed in this paper.
} 
section. Moreover, it explains why our actual candidates for the physical laws have several striking features that make them useful for creatures like us.

Our version of the BSA makes it an explicit requirement on laws of nature that they are useful for creatures like us. In particular, we defend the following constraint on laws:

(CU) Laws of nature encode information in a way that maximizes their cognitive usefulness for creatures like us. ${ }^{10}$

By 'creatures like us', we mean creatures with limited cognitive resources (cf. Caton 2014 and Morton 2012). We do not mean specifically humans or humans given our current state of technology. We mean creatures that have limitations with regard to their cognitive resources that are general enough to be shared by all embodied agents, including current humans, future humans, and alien scientists (if there are any).

These limitations include the following: First, finite beings only have incomplete information. They never know the state of the world with full precision. Instead, their knowledge will be partial and possibly slightly inaccurate due to the limitations of their perceptual faculties and measurement equipment. Second, they are limited with regard to how much information they can store and process. In particular, limited creatures cannot compute arbitrarily complex functions. And, third, limited beings have finite resources. So, they have an interest in completing cognitive tasks as efficiently as possible, that is, by minimizing how much information they need to gather, store, or process. Our proposal is that scientific inquiry is structured around the needs of creatures with these kinds of limitations.

The other notion that needs clarification is 'cognitive usefulness'. We assume that the main cognitive function of the laws is facilitating predictions. Laws of nature allow us to derive new truths from known truths. The laws plausibly have further cognitive functions, such as action-guidance and explanation. But we will focus on prediction because it arguably is the function of the laws that is most central to the practice of physics. So, CU says that laws of nature help limited beings get around in the

\footnotetext{
${ }^{10}$ You may worry that CU is objectionably anthropocentric, but we will argue below that the ensuing anthropocentricity is unproblematic.
} 
world by encoding truths that help them make predictions from information available to them.

We propose a modified BSA that incorporates CU. Best systems need to be best for $u s$, that is, contain information that is useful for solving the kinds of 'predictionproblems' that limited beings face. These problems involve predicting the evolution of physical systems from incomplete and possibly slightly inaccurate information. A system that is best for us should facilitate solving as many of these problems as possible with as little cognitive effort as possible. Such a system has to allow us to make the relevant predictions from the available input without being too demanding on our capabilities for storing and processing information. We propose that the laws are whatever facts are part of such a best system. ${ }^{11}$

Some examples will help illustrate what such systems look like. Suppose you want to predict the future behavior of a rock on your desk. First, consider a system that requires a complete description of the world's current microstate as input. Such a system would not be cognitively useful for limited beings because they do not possess such complete information. Second, consider a system that just lists every particular truth about the world. This system would not require any input about the current state of the world for making predictions about the behavior of the rock since all information is already part of the system itself. However, it would be massively resource-intensive to store all of this information and to locate the relevant bits about the behavior of the rock. So, this system also is not cognitively useful. Finally, consider a system that is relatively easy to store (perhaps a small number of differential equations) and that allows you to predict the future behavior of the rock when combined with (approximate) information about its current state. Such a system would be enormously cognitively useful since it is relatively easy to store and the required input readily available and easy to process.

Systems that are best for us need to balance competing virtues. First, they need to minimize the complexity of the laws. Systems that are highly complex (a long list of particular facts) are more difficult to process than less complex facts (a small number of differential equations). Second, cognitively useful systems need to minimize the input

\footnotetext{
11 Ismael (2015: 197) proposes that laws are "partially prepared solutions to frequently encountered problems." Our account can be seen as a development of this idea. (Ismael herself does not wholeheartedly endorse Humeanism about laws.)
} 
information' that is needed to make predictions (cf. Sober 1975 and Thagard's 1978: 88). For example, systems that allow us to predict the future evolution of a rock from its rough macroscopic state are easier to apply by limited beings than systems that require as input its exact microstate. These two virtues can trade off against each other. Sometimes by building more information into the laws, less information about the background conditions is needed to use them for making predictions. For example, a system of laws that already has all information about the rock built into it requires no added information about background conditions to make predictions. But building this information into it comes at the cost of making the system very complex and difficult to process. A system will be more useful the less information you need as input and the less storing and processing is required to arrive at the relevant predictions. Systems that are best for us will be relatively simple, yet be combinable with incomplete and possibly slightly incorrect information about the background conditions to result in many predictions.

Best systems that are best for us are different from Lewis's best systems in at least two respects. First, Lewis's best systems aim to provide as much information as possible about the entire Humean mosaic in as simple a form as possible. Our best systems, by contrast, do not aim to provide this information all by themselves. Instead, they aim to provide the kind of information that limited beings can efficiently use to infer from truths about certain bits of the mosaic to truths about other bits of the mosaic. Second, Lewis's best systems adhere to objective global virtues of strength and simplicity. By contrast, the virtues of our best systems are tailored to the capacities of cognitively limited beings.

We will defend our account by arguing that CU is an independently plausible constraint on laws of nature. If cognitive usefulness is a virtue of laws of nature, we expect the information encoded in the laws to be streamlined toward making predictions from incomplete information. We will provide two arguments that show that the laws science aims for have exactly this form.

Our first argument for $\mathrm{CU}$ is that our best past and present candidates for the fundamental physical laws have features that make them cognitively extremely useful. This point is not obvious. Our best candidates for the fundamental laws since at least Newton are such that what happens in one place may depend on what happens in numerous other places. Newtonian gravitation and classical electromagnetism, for 
example, entail that every massive or charged body influences every other massive or charged body. Things get slightly better with Special Relativity, but the behavior of a system may still depend on what happens in a cross-section of its entire backward light cone. Moreover, these laws are such that the exact microstate of a system can in principle make a difference to its macroscopic evolution. So, it seems that using the actual laws to predict the evolution of any system would require more information than creatures like us will ever have or be able to compute.

However, our best candidates for the laws of nature have a variety of features that allow us to derive approximate truths about systems from incomplete information. First, these laws have a high degree of 'error tolerance' (cf. Callender 2017: ch. 7). For many problems, the solutions depend on the data continuously such that small errors in the data only lead to small errors in the solution. For example, suppose you want to predict the trajectory of a (point) mass that is subject to a constant net-force. Solving the differential equations you get from Newton's second law tells you that the future positions of your mass is among other things a continuous function of its initial position. Thus, a small difference in its initial position only makes a small difference to its future position. ${ }^{12}$ This error-tolerance allows that the laws can be used to derive approximate truths despite inaccuracies in the available data.

Second, as Elga (2007) shows, our laws are such that, given a small number of background assumptions, they do allow predictions about systems from relatively local bits of information (see also Ismael 2009: 91-92 and Loew 2017). Suppose you want to predict the future evolution of a rock on your desk. Elga points out that almost all of "the forces acting on your rock from afar are either negligibly tiny or nearly constant" (Elga 2007: 109). Physics describes four types of forces. Of these forces, strong and weak nuclear forces are negligible over distances that are large compared to the atomic scale. Electromagnetic forces can be strong across larger distances, but there are not very many strongly charged macroscopic bodies around. So, it usually is negligible too. Finally, gravitation can be strong across large distances. However, the net gravitational force on objects usually does not vary significantly across time and space. For example, the net gravitational force on a rock on your desk yesterday is roughly the same as the net gravitational force on a rock on my desk today. So, whenever we predict the

\footnotetext{
12 For chaotic systems, however, small inaccuracies in the data do lead to big inaccuracies in the solutions even if dependence is continuous.
} 
evolution of a rock, we can typically treat the forces acting on it from afar as constant apart from small variations.

We then only need to guarantee that these small variations in background factors (at least in the majority of cases) do not make a difference to the rock's macroscopic behavior. Fortunately, this result follows from features of the dynamical laws and plausible background assumptions. Our best candidates for the fundamental dynamical laws are such that for many macroscopic system the vast majority of compatible microstates are associated with the same macroscopic behavior (this feature is closely related to the above described error-tolerance). We then only need to assume that a given macroscopic system is in such a 'typical' microstate. This typicality then guarantees that small differences in external forces are unlikely to make a difference to its macroscopic behavior. Small difference in outside forces only make a difference to the system's microstate. But since the vast majority of microstates compatible with a given macrostate are associated with the same macroscopic behavior, such forces do not result in a different macroscopic behavior (cf. Elga 2007: 110). So we can ignore any forces acting on the rock from afar that are so small that they only affect its exact microstate, such as air molecules bumping into it or small local variations in gravitational forces.

It is a highly non-trivial feature of our best candidates for the fundamental dynamical laws that the vast majority of microstates compatible with a given macrostate lead to the same macroscopic behavior. And it is empirically well-confirmed that we usually get the correct predictions about the future behavior of systems by assuming that their behavior is 'typical'.13 These two facts together allow us to describe the behavior of systems in terms of autonomous, higher-order dynamics, as they feature, for example, in thermodynamics. As a consequence, coarse-grained information about the rock plus the knowledge of a small number of background conditions is enough to predict its rough macroscopic evolution. These features of our actual best candidates for the laws of nature then are evidence that science aims for laws that are cognitively useful for creatures like us.

13 There is considerable debate in the foundations of statistical mechanics about what the assumption that a system's behavior is 'typical' involves. See Frigg (2009) for discussion. See Albert $(2000,2015)$ for a similar account. 
Our second argument for CU is based on how scientists use the laws of nature in practice. Philosophers tend to think of the fundamental dynamical laws as operating on fully-specified global time-slices. But, as Frisch points out, physicists often use the laws to make inferences from 'partial models', that is models that only provide an incomplete, coarse-grained specification of the relevant circumstances:

Very often, even within the context of established or putatively "fundamental" theories, we represent phenomena not in terms of a complete initial- and boundary-value problem, which would provide us with information about the precise physical state of each spacetime point in the spacetime region of interest, but by specifying only a finite number of relevant components of a system at different levels of grain and then showing how these components interact. (Frisch 2014: 62)

Frisch's example is the Large Hadron Collider. When physicists model proton beams inside the collider, they leave out many outside influences on the accelerator and model other components only at a very coarse-grained level (see Frisch 2014: 63).

Partial models are crucial for physical practice because they allow physicists to apply the laws of nature to real life physical systems. The laws, at least in principle, allow derivations from fully specified microstates. But such derivations would not allow us to make any real-world predictions because we cannot know whether the precise microstates from which such predictions are derived actually obtain. As Frisch points out, "the models physicists in fact use to represent actual phenomena very rarely if ever are the complete structures that philosophers postulate, because these structures are in general much too complex for us ever actually to possess" (Frisch 2014: 66). In applying the laws to partial models, we make predictions about situations that are specified rather coarsely. We can then be reasonably sure that these situations obtain at least in carefully prepared experimental set-ups, and so test the relevant predictions.

Not all conceivable laws of nature would allow the successful use of partial models. We can imagine laws whose predictions are so sensitive that one needs to know the complete microstate of the universe at some time to derive any reliable predictions. For example, suppose a set of putative laws is such that a difference in the position of any distant particle would result in radically different predictions about the macroscopic evolution of systems. Such laws could not be used to derive predictions about systems by applying them to partial models. 
It is a highly significant feature of our actual candidates for the fundamental laws that they are applicable to partial models. Partial models specify physical situations in an incomplete and coarse-grained way, which is crucial for allowing us to know when the relevant models hold true of real life situations. So, laws that promote (approximately) true predictions based on partial models need to license derivations from incomplete information. And, as illustrated above, this condition is a defining feature of laws that satisfy CU. Hence, the same laws that are easily applicable to partial models also satisfy CU. The centrality of partial models to physical practice then shows that physics requires laws that are cognitively useful in the sense of CU. We conclude that physical practice provides strong evidence for CU.

(There are several other features, in addition to error-tolerance and allowing the use of partial models, that make actual candidates for the laws cognitively useful for us. Let us briefly mention a few more: First, the laws exhibit striking symmetries. If the laws varied widely depending on where and when scientists perform experiments or on how the target system is spatially oriented, they would be virtually useless for us. Second, non-classical laws (e.g., the laws of Special Relativity) often allow the use of (mathematically) much simpler classical laws (e.g., Newton's laws of mechanics) as 'limiting cases'. Third, our best candidates for the laws are Markovian such that we can predict what happens to systems from the state of the world at a slightly earlier time without having to consider what happened (for instance) in the distant past and usually allow predictions from 'spatially local' information. And, fourth, the actual candidates for the laws all are expressed by computable functions (see Deutsch 2012)).

In the rest of this section, we will address two possible worries. A first worry about CU is the resulting anthropocentricity. CU entails that the standards for lawhood implicit in science are ultimately rooted in our situation as limited beings. Lewis called the worry that the laws according to the Humean BSA are objectionably anthropocentric the "problem of the ratbag idealist" (see Lewis 1994: 476 and Hall 2015: 267-269). Note that CU does not imply that what the laws say about the world is relative to our cognitive capacities. For example, if Newton's equations come out as laws, then the fact that particles behave in accordance with Newton's equations is a truth about the Humean mosaic that is in no way relative to our cognitive capacities (cf. Cohen and Callender 2009: 30). CU, however, does entail that which facts about the Humean mosaic have the status of laws is partly explained by our cognitive capacities. And which 
facts are laws, according to Humean reductionism, then helps determine the world's counterfactual, causal, and dispositional structure.

In reply, we argue that CU's anthropocentricity is not objectionable. First, the standards of lawhood according to our view are non-voluntary and robust. The only fact about us that matters to what the laws are is that we have limitations with regard to gathering and processing information. These limitations are independent of what we believe or desire. So, the laws are in no way 'up to us'. Moreover, these limitations are shared by all agents we know and are not likely to change over time. While technology may improve significantly, there will still be limitations on how much we know and can process. Our view can then explain how the scientific community can disagree about what the laws are and make progress. There can be objective disagreement about what information is most cognitively useful for limited beings. And yet to be discovered best systems may be cognitively much more useful for limited creatures than any current candidates for the fundamental laws. ${ }^{14}$

Second, the resulting anthropocentricity nicely accounts for certain features of scientific practice. We have shown above that many de facto features of laws, such as error-tolerance or applicability to partial models, are to be expected if science aims for laws that are cognitively useful for creatures like us. So, our account explains why our best candidates for laws have these features. By contrast, non-anthropocentric theories that construe laws as independent of the capacities of creatures like us appear to have no explanation for why the laws have these and other remarkable features that make them cognitively useful for creatures like us.

Third, Humean reductionism is more plausible as a result of being anthropocentric (cf. Loewer 2007: 325). Its limited anthropocentricity allows Humeans to make do with a very sparse ontology because no extra metaphysical structure is needed to distinguish laws from non-laws. Bringing in our situation as agents nicely explains why certain facts about the world are of distinctive interest to our inquiry but not others. By contrast, theories maintaining that the standards for lawhood are completely independent of our situations as cognizers inevitably need to posit additional ontological or ideological machinery that singles out the laws. Letting our

\footnotetext{
${ }^{14}$ What if there are alien scientists who, like Gods or Laplacean demons, can know the world's state with complete precision and have no limitations in their processing power? We contend that these agents, if they have a concept of a law of nature at all, would have a different concept from ours such that our laws would not be of particular interest to them.
} 
make-up as cognizers do this work dispenses with this machinery and has the additional benefit of making immediately clear why we care about the distinction between laws and non-laws. ${ }^{15}$ In light of these three points, the anthropocentricity arising from making best systems best for us is not objectionable.

The second worry is that CU may be at odds with certain prime candidates for fundamental physical laws. In particular, you may think that the laws of quantum mechanics do not seem geared toward being cognitively useful for creatures like us. After all, the interpretation of these laws is notoriously tricky and we can at best derive probabilistic predictions from them. So, you may worry that the laws of quantum mechanics would, according to CU, not be good candidates for fundamental physical laws.

In reply, we argue that science's preference for the laws of quantum mechanics is fully in accordance with CU. First, quantum mechanics' core formalism, consisting of the quantum state, the Schrödinger equation and the Born rule, allows us to derive a large number of predictions from incomplete information. Quantum mechanics is our empirically best confirmed theory in the history of science. So, actual limited scientists have derived more accurate predictions from it than from any other theory. Indeed, quantum mechanics has features similar to the features that make classical mechanics useful for limited beings. Macroscopic systems can often be treated as relatively isolated from their surroundings even in cases where locality is violated due to quantum entanglement (see Elga 2007: 109, fn. 7). And, moreover, quantum mechanics allows us to make predictions even when we are ignorant of the exact microstate of the relevant system. We can often, at least in practice, derive the approximately correct predictions about macroscopic systems in terms of higher-level dynamics that allows us to ignore various aspects of the relevant systems (see Wallace forthcoming: §10). ${ }^{16}$

Second, that science aims for laws that are cognitively useful does not mean that the resulting laws will be perfectly tailored to our cognitive needs. Nature, after all, needs to cooperate too. We can imagine candidates for the fundamental physical laws that would be more useful for limited creatures than the laws of quantum mechanics.

\footnotetext{
${ }^{15}$ Lewis's effort to make his version of the BSA non-anthropocentric, by contrast, comes at the cost of positing perfectly natural properties (Lewis 1983: 367-368) and objective standards of strength and simplicity (Lewis 1994: 479). However, it is then not clear that scientists should accommodate this structure when discovering laws. See Cohen and Callender (2009: sec. 2.1) and van Fraassen (1990: 53) for criticisms along these lines.

16 Thanks to David Glick for discussion of this point.
} 
For example, laws that are completely deterministic. (In quantum mechanics, the Schrödinger equation is deterministic, but it is hard to derive predictions from it without the Born rule, which only yields probabilities of measurement outcomes.) But we also want laws that allow us to make as many predictions as possible. And quantum mechanics, given the abundance and accuracy of its predictions, is the most cognitively useful theory we currently have. So, the success of quantum mechanics is fully compatible with the truth of CU.

\section{Why best for us is best}

In this section, we will argue that incorporating CU into the BSA enables a reply to Hall's challenge because it provides a Humean motivation for why the laws display modal latitude. A central feature of cognitively useful laws is their robust applicability. Cognitively useful laws allow the derivation of (approximate) truths from incomplete and even inaccurate information. So, these laws are robust with respect to how complete and accurate information about systems needs to be to facilitate the derivation of (approximate) truths. This robustness allows creatures like us to apply the laws to actual physical systems because our knowledge of these systems and their environment is always incomplete and often slightly inaccurate.

Modal latitude is a byproduct of robust applicability. Think about what is involved in using the laws for making inferences from incomplete and inaccurate information. There are two general options: First, you can apply the laws to a partial model of the system that leaves out features about which you lack precise information. For example, we may make predictions about a collision between two proton beams from a model that omits various distant objects. This partial model then represents nonactual circumstances in which there are no such distant objects. Second, you can specify precise values for all physical parameters and then apply the laws to the so-specified model. But since we are almost surely mistaken about the exact values of many parameters, this model will also almost surely represent non-actual circumstances. You might also use a 'mixed approach' where you leave out some facts and represent others somewhat inaccurately. Either way, making inferences from such information requires applying the laws to non-actual circumstances. Laws that are only applicable to the actual situation are useless when you lack precise knowledge of the relevant actual 
circumstances. So, modal latitude is a necessary condition for the laws' allowing inferences from incomplete or slightly inaccurate information.

Applying the laws to non-actual circumstances in this way is purely in the service of making (approximately) true predictions about actual systems. If the laws are cognitively useful, then we often can make (at least approximately) true predictions about actual systems by applying the laws to non-actual circumstances. For example, Elga (2007) points out that, according to our actual candidates for the laws, the forces exerted by many distant objects typically do not matter to the macroscopic behavior of systems. So, we can derive approximately true predictions about the actual behavior of macroscopic objects by applying the laws to non-actual circumstances in which these distant objects are absent.

Moreover, applying the laws to a range of non-actual circumstances tells us what we can neglect when making predictions about actual systems. Suppose you apply the laws to a range of situations that differ with respect to the exact masses of distant objects. If certain predictions follow given all (or at least most) mass values, then you can be reasonably sure that the exact masses of distant objects do not matter for these predictions. This procedure, however, presupposes that the laws are applicable to nonactual circumstances in the first place. Similarly, our best candidates for the physical laws are 'error tolerant' in the sense that small mistakes in the data often only lead to small mistakes in the solutions (see §3). So, we can derive (approximately) true predictions about actual systems by applying the laws to slightly inaccurate models.

According to our Humean account, modal latitude is a byproduct of the laws allowing us to infer (approximate) truths given our epistemic situation as limited creatures. Humean best systems that narrow down the range of possible initial conditions to a too small set of non-actual scenarios are deficient not because they ignore some genuine modal aspect of fundamental reality. They are deficient because they prevent us from using the laws for making inferences about actual systems from incomplete or inaccurate information and so are cognitively useless. Applying the laws to non-actual scenarios thus serves the arch-Humean aim of collecting information about the actual non-modal Humean mosaic.

Our perceptual faculties are limited such that we can never know the state of the world at a given time with complete precision. So, we cannot rule out the epistemic possibility that certain non-actual circumstances obtain. The laws then need to allow 
these epistemic possibilities as nomological possibilities in order to allow us to infer (approximate) truths about the state of the world at other times from our available evidence. So, information the laws tell us about what would have happened if non-actual circumstances had obtained is not sui generis. It is information about the actual mosaic that allows us to make inferences from fragmentary inputs. Thus, according to our account, the laws' modal content is ultimately due to our epistemic ignorance.

A nice illustration of how our Humean account explains the modal latitude of laws is the resulting story of why functional laws, such as Newton's second law, range over values that are plausibly never instantiated in the actual world. By Humean lights, it seems puzzling why science prefers laws that have such excessive, apparently modal content over laws that range only over values that are actually instantiated. What Humean reasons are there for preferring laws that cover uninstantiated values?

A traditional answer is that unrestricted laws are simpler because excluding uninstantiated values would prevent us from writing down, e.g., Newton's second law as a simple equation (see Armstrong 1983: 22-23). We think that there is something right about this response because simpler laws are easier to use in calculations and so are more cognitively useful. But our need to use laws for making inferences given incomplete and inaccurate information provides two new reasons for preferring laws that cover uninstantiated instances.

First, due to measurement errors, we are often mistaken about the exact values of observed quantities. Since many laws are such that small mistakes in the data often only create small mistakes in the solution, we still can derive useful predictions from such inaccurate data by applying the laws to non-actual values. And, second, we often know only the approximate values of certain physical quantities. It is then enormously useful if we can apply the laws to a wide range of precise values that are all compatible with our epistemic state. If certain predictions hold true for all or most of these epistemically possible values, then we can be reasonably confident that they are true for the actual system. ${ }^{17}$ In this and the previous case, it is enormously useful that the laws cover uninstantiated instances because it allows us to make approximately true predictions given incomplete or inaccurate data.

We have shown how Humeans have an account for why laws apply to non-actual circumstances. But there is still a question of why scientific laws apply to the range of

17 Thanks to Mike Hicks for discussion of this point. See also our discussion of Elga in $§ 3$. 
non-actual circumstances to which they in fact apply. Why does physics regard some circumstances as nomologically possible but not others? This question is equivalent to the question of why science treats certain facts as laws, such that they hold in all nomologically possible worlds, but treats other facts as mere boundary conditions. A Humean theory needs to explain why, for example, facts like Hall's phony constant are not serious candidates for laws of nature.

CU explains why Hall's phony fundamental constant is not a candidate for a law of nature because it is not part of best systems that maximize cognitive usefulness. The phony constant is a single real number that encodes the entire state of the universe at an instant. It is created by interleaving the exact numerical values of the coordinates, masses, and charges of every single particle at that instant. Limited beings cannot grasp the exact value of the constant. However, grasping the phony constant only approximately does not help you to make any predictions, since that does not mean that you will know the approximate positions, masses, and charges of all particles (Hall 2015: 273-274 attributes this response to Barry Loewer).

Here is a brief illustration of this last claim: Suppose you have a list of the exact values of the masses, charges, and spatial coordinates of every single particle in the universe at an instant $t$. A natural way of encoding all of this information into a single real number is by interleaving for every single particle its mass-value, its charge-value and the values of the spatial coordinates into a single number. Then you take all of the resulting numbers and interleave them again to get a single number, which now encodes the complete state of the universe at $t$. If you know only the first thousand digits of this number, all you know is the first digit of, say, the mass-value of each of a thousand particles. This information hardly helps you make any interesting predictions. This feature distinguishes the phony constant from legitimate constants, such as the gravitational constant $G$. Plugging an approximate value for $G$ into the gravitational law is good enough to determine the gravitational forces between any two masses at least approximately. So, in contrast to the phony constant, $G$ contributes to cognitive usefulness of the laws because even its approximate value is very useful for making predictions.

And even for a creature with enormous computational powers, who could store and process the exact phony constant, the maximal strength that deterministic laws including the phony constant provide would come at the cost of extreme inefficiency. 
For instance, a rock's rough macroscopic evolution can be predicted from coarsegrained information about it and a small number of its background conditions. If this evolution is instead derived from the dynamical laws and the phony constant, the agent needs to process all the extra information stored in the phony constant that is not necessary to make the prediction at hand.

Worse, if we cannot process the exact value of the phony constant, then adding it to a set of deterministic dynamical laws might even drastically decrease the predictions a limited creature can make. The constant, in combination with deterministic laws, narrows down the range of possible initial conditions to just the actual ones (as illustrated above). Thus, the resulting set of laws would not be applicable to partial and slightly erroneous models. These models represent different initial conditions from the one of the actual universe, and so they are ruled out as nomologically impossible by the phony constant in combination with deterministic laws. But a set of laws that we cannot use to make inferences from incomplete information is virtually useless for creatures like us.

Making best systems best for us rules out other implausible candidates for laws of nature in a similar way. ${ }^{18}$ Hall objects that many facts that would be enormously useful to know are still not candidates for fundamental physical laws. For instance,

cosmologists would very much like to know the total mass of the universe - and [...] even approximate information about this mass would be enormously predictively and explanatorily valuable. But would their ability to squeeze useful predictive and explanatory information out of this knowledge do anything to show that facts about the total mass should be viewed as nomologically necessary? (Hall 2015: 274)

It seems that even knowing only the approximate total mass of the universe might be extremely useful for deriving certain predictions. A theory of laws of nature should explain why the mass of the universe is still not a candidate for a law.

${ }^{18} \mathrm{CU}$ also solves a famous problem for Lewis's version of the BSA. Take a maximally strong system $S$ and "[l]et $F$ be a predicate that applies to all and only things at worlds where $S$ holds. Take $F$ as primitive, and axiomatize $S$ (or an equivalent thereof) by the single axiom $\forall x F^{\prime \prime}$ " (Lewis 1983: 367). Since $\forall x F x$ is maximally informative and simple, Lewis's BSA has the absurd consequence that all regularities whatsoever come out as laws. Lewis evades this problem by restricting predicates to ones referring to perfectly natural properties. Our account requires no such objective joints, since $\forall x F x$ does not provide information that is storable or computable by resource-bounded creatures and so is cognitively useless. Our account, thus, incorporates the central insight of the so-called 'Better Best System Account', viz., that Humeans need not (and should not) posit a naturalness constraint on the language the best system is couched in (see Cohen and Callender 2009). 
In reply, we argue that including the mass of the universe in a best system would decrease its cognitive usefulness. As Hall points out, knowing the mass of the universe is very valuable for certain predictions. For example, it might be useful for deriving whether the universe will expand forever or end up in a big crunch. However, suppose you want to know how a rock on your desk evolves over the next two minutes; whether a certain bridge will survive an upcoming storm; or what happens when two proton beams meet in the Large Hadron Collider. To solve these and similar problems, it is not necessary to know even the approximate mass of the universe. Consequently, the mass of the universe would add information to the laws that is completely irrelevant for most (approximate) truths we derive from the laws. Thus, the rather minimal increase of predictions that adding the mass of the universe adds to the laws provides comes at the price of decreasing their efficiency significantly. So, it is more useful to treat the mass of the universe as an auxiliary hypothesis that is called on when needed rather than to add it to your laws. ${ }^{19}$ (Notice that a system that balances strength and simplicity as Lewis understands them may rule out the phony constant for similar reasons.)

A similar worry about our account concerns why our best candidates for laws of nature allow possible initial conditions where creatures like us do not exist, for example where there is no life in the universe. If the laws are tailored toward helping creature like us navigate the world, why should they be applicable to situations that exclude the existence of such creatures? We reply that adding conditions that ensure the existence of creatures like us to the laws would add extra information to the laws that are irrelevant for most predictions. For example, when we want to predict the evolution of a rock, it is irrelevant whether the universe contains life or not. Moreover, if we want to use the laws to make predictions from the kind of evidence creatures like us have, then it would be redundant to build into the laws that creatures like us exist. Our having of the evidence already entails that there is life in the universe.

\footnotetext{
19 You may worry that the above considerations also rule out certain bona fide laws. Suppose (as may well be the case) that weak nuclear force is completely irrelevant to most of the approximate truths that we derive from the laws. Why then should there be a force law for that force? What is the difference between that force law and a fact about the approximate mass of the universe? In reply, we argue that we still would need a force law to tell us that weak nuclear force can be ignored in almost all situations. With regard to the approximate mass of the universe, the gravitational laws tell us that we can ignore it in most cases. But without a law for weak nuclear force, we would have no idea whether in any given situation, we could ignore weak nuclear force or have to take it into account after all.
} 
CU also explains why facts that decrease the modal latitude of the laws (by making them apply to fewer non-actual circumstances) sometimes can be candidates for laws of nature. For example, Albert (2015) proposes adding two additional fundamental laws to our best physical theories: the "past hypothesis," which says that "the universe had some particular, simple, compact, symmetric, cosmologically sensible, very low-entropy initial macrocondition" (Albert 2015: 5). And the "statistical postulate," which specifies a uniform probability distribution over the microstates compatible with the past hypothesis (Albert 2015: 5). Regarding the past hypothesis as a fundamental physical law reduces the modal latitude of the laws because it rules out possible initial conditions where the universe started in a different macrocondition than its actual, low-entropy one.

CU explains why the past hypothesis and the statistical postulate may be candidates for laws of nature. ${ }^{20}$ According to our account, science aims for modal latitude only to the extent that doing so facilitates inferring (approximate) truths from incomplete or inaccurate information about systems. If applying the laws to non-actual circumstances is not useful for this purpose, it is no longer a virtue for the laws to cover these circumstances. Now, adding the past hypothesis to the laws rules out initial conditions where, for example, the universe started in a high-entropy macrostate. Covering these possible initial conditions, however, is not required to make the laws applicable to the incomplete or slightly inaccurate models that we typically use for predictions. These models are plausibly still compatible with the initial macrocondition of the actual universe, and so the past hypothesis does not get in the way of allowing us to derive (approximate) truths from incomplete or inaccurate information.

If Albert is right, then the past hypothesis and the statistical postulate are in fact required to derive (approximate) truths from incomplete information. Dynamical laws alone, even deterministic ones, are not enough if you do not know a system's state with complete precision. All plausible candidates for the dynamical laws allow, given only a macroscopic description of a system, multiple different behaviors, many of which would strike us as bizarre. For example, if you consider a rock governed by the Newtonian laws of motion, then nothing "is going to stand in the way of that rock's suddenly ejecting one of its trillions of elementary particulate constituents at enormous speed

${ }^{20}$ We suspect that something similar applies to other candidates for fundamental physical laws that decrease modal latitude, such as force laws and other laws of coexistence. 
and careening off in an altogether different direction, or (for that matter) spontaneously disassembling itself into statuettes of the British royal family, or (come to think of it) reciting the Gettysburg Address" (Albert 2015: 1). So, deriving any approximately true predictions from incomplete information requires a probability distribution over microstates that makes sure that such bizarre behaviors have negligibly small probabilities (cf. Ismael 2009: 91-92). (Note that such a probability distribution is also relevant for why we can largely ignore distant influences when making predictions about the macroscopic behavior of systems. See our discussion of Elga in §3).

Albert argues that the past hypothesis and the statistical postulate specify the needed probability distribution in a very simple way. If Albert is right about this role, then adding the past hypothesis and the statistical postulate to our laws dramatically increases the laws' cognitive usefulness because it is necessary for efficiently deriving (approximate) truths from incomplete information. ${ }^{21}$ This radical increase in the number of predictions one can make from incomplete information would then plausibly outweigh the added complexity. This role distinguishes the past hypothesis and the statistical postulate from, e.g., the mass of the universe. While knowing the mass of the universe is occasionally required for deriving certain (approximate) truths, the former facts, if Albert is right, are relevant whenever we derive any (approximate) truths from mere macroscopic information about a system.

That facts like the past hypothesis are candidates for laws of nature is evidence that science does not aim unconditionally at a strong DH and a weak ICH in Hall's sense. Recall that the ICH measures how many possible initial conditions a set of laws allow and the DH measures how many possible ways there are for the world to evolve given specified initial conditions. Adding the past hypothesis and the statistical postulate to deterministic dynamical laws does not strengthen their DH. So, deterministic laws already have as strong a DH as laws can possibly have.

But adding the past hypothesis and the statistical postulate does strengthen the ICH because it rules out metaphysically possible initial conditions that are allowed otherwise. Focusing on cognitive usefulness explains why we still consider these facts as candidates for laws of nature. Allowing a certain range of possible initial conditions is

\footnotetext{
${ }^{21}$ This "if," however, is a big "if." It is controversial both whether the past hypothesis and the statistical postulate really provide the required information and whether they do so in the most efficient way. See Albert (2015: 6, fn. 2) and Callender (2011) plus the references therein for discussion.
} 
useful because it allows us to apply the laws to system of which we have incomplete or inaccurate information. But ruling out other initial conditions can also be useful if it allows us to ignore certain dynamically possible behaviors when making inferences from incomplete information. Playing this latter role makes the past hypothesis (in conjunction with the statistical postulate) a candidate for a law of nature despite decreasing modal latitude.

Summing up the results from the previous two sections, our version of the BSA has the following three benefits: First, it is in accordance with scientific practice because actual candidates for the laws of nature have features that make them enormously cognitively useful for creatures like us. Second, it provides a Humean motivation for why scientific practice aims for laws that cover a range of non-actual circumstances. And third, it explains why certain restrictions on the initial conditions (such as the past hypothesis) are candidates for scientific laws while others (such as the phony constant) are not.

\section{Other Humean accounts of modal latitude}

In the remainder of the paper, we want to contrast our account with other recent Humean accounts that also emphasize the laws' usefulness for limited beings. The guiding idea of Hicks's (forthcoming) "Epistemic Role Account" is that the laws are those facts that are constant between subsystems. Hicks emphasizes that the laws of nature need to be discoverable and confirmable by limited beings. Limited beings lack access to the state of the universe as a whole, and so laws that are confirmable by limited beings need to apply to subsystems. The rough idea is that if we consider some actual subsystem, such as our solar system, and treat it as if it is completely isolated from its environment, then the laws should describe that system approximately correctly. Moreover, the laws are easier to confirm if they apply to as many actual subsystems as possible in this way and if they make many accurate predictions about each system. ${ }^{22}$

Hicks's proposal is similar to our account because it plausibly is part of the laws' cognitive usefulness that limited beings can find out about them and confirm them. Hicks is less concerned than we are with providing a Humean motivation of why science

\footnotetext{
${ }^{22}$ Hicks calls the corresponding constraints "local strength" and "breadth." He proposes other interesting constraints on laws, but these are not relevant for the discussion at hand.
} 
entertains the standards of lawhood it in fact entertains. His main goal is to present a Humean account that correctly demarcates the laws from the non-laws. In particular, he wants to explain why facts such as Hall's phony constant are not laws but boundary conditions. Nonetheless, Hicks's account provides a Humean motivation for the laws' modal latitude. Laws that are easily confirmable by creatures like us, according to Hicks, need to apply to many different subsystems when these systems are treated as if they are isolated. And since subsystems in our universe are never in fact completely isolated from their environment, the relevant circumstances are non-actual. So, Hicks's proposal explains why Humean laws apply to non-actual circumstances.

Hicks's account, however, lacks the same independent motivation as our account. First, making predictions about many actual subsystems is at most necessary for the laws' cognitive usefulness but not sufficient. Laws that satisfy Hicks's requirements may still not allow creatures like us to make predictions. Suppose you want to predict the future evolution of a rock on your desk. Hicks's restriction on laws guarantees that the laws make the correct predictions for as many subsystems as possible if these systems are treated as if they were isolated. But laws that satisfy this restriction may still give you no guidelines for knowing whether they will make the correct predictions about this particular rock if you treat it as if it is isolated. Moreover, even if the laws do make the correct predictions about the rock treated as if it is isolated, limited creatures may not be able to derive these predictions. Limited beings are not just ignorant of the rock's environment but also of its exact microstate. So, even laws that make the correct predictions about the rock if it is treated as if it were isolated are still not useful for creatures like us if they require as input its exact microstate. Yet, Hicks's restrictions on laws by themselves do not entail that the laws allow approximately correct predictions from partial information about subsystems.

For laws to be cognitively useful for creatures like us, the laws need to guide us with regard to when we can and cannot treat subsystems as if they are isolated from everything else and still get the approximately correct predictions. For example, Newtonian laws tell us that when we make predictions about a rock on Earth, we can ignore most objects in its surrounding. However, they also tell us when we cannot ignore them, such as when the respective objects are extremely massive. Moreover, for the laws to allow us to make predictions given our ignorance of the rock's exact microstate, they need to be applicable to all epistemically possible microstates of the 
rock and tell us that typical such microstates have the same macroscopic evolution. Only given this information are we justified in making any predictions. So, while Hicks's account incorporates important insights, facts that satisfy Hicks's requirements may still not be cognitively useful for creatures like us.

Second, a more technical worry about Hicks's account is it that it is not clear whether it can allow non-dynamical laws that would restrict the boundary condition of the universe. For example, the past hypothesis specifies that the early universe "had some particular, simple, compact, symmetric, cosmologically sensible, very low-entropy initial macrocondition" (Albert 2015: 5). If the past hypothesis is a law, then it seems to drastically reduce the number of subsystems to which the laws apply. This law, by definition, only seems to apply to the whole universe since it references its initial macrostate. And even if it could somehow be adapted to smaller systems, it will not be true of many of them. For example, many subsystems taken by itself, such as a gas that is evenly spread out in a box, are not in states of very low entropy. So, Hicks's account rules out facts such as Albert's past hypothesis as candidates for laws of nature. By contrast, our Humean account of laws can make room for facts like the past hypothesis as candidates for laws because they may increase the laws' cognitive usefulness for limited beings (see $\S 4$ ).

Another recent account that appeals to the cognitive usefulness of best systems is Cohen and Callender's (2009) "Better Best Systems Account." Cohen and Callender reject Lewis's restriction that the predicates in best systems need to refer to perfectly natural properties. Instead, they allow many different sets of predicates and propose to run a separate bestness competitions to determine the best systems relative to each choice of predicates. A constraint analogous to cognitive usefulness then comes in because when faced with the question of which of these various best systems to choose, they appeal to our "pragmatic/explanatory goals (rather than the fixed nature of the universe)" (Cohen and Callender 2009: 21).

But Cohen and Callender appeal to these pragmatic factors only with regard to choosing the predicates in terms of which best systems are framed. They play no role for explaining the criteria that decide, relative to any choice of predicates, which system is best. Our above argument, however, shows that appeal to practical concerns, in the form of what information is useful for limited agents, is already needed to motivate why 
science uses the standards of lawhood it in fact uses and, in particular, why these standards favor laws with modal latitude. ${ }^{23}$

\section{Conclusion}

We have spelled out an important challenge for Humean reductionism about laws of nature and motivated a new version of the Humean BSA that meets this challenge. We have argued that the best way of understanding the BSA is by acknowledging that the laws of nature are tailored toward helping limited beings make predictions from information accessible to them. This take on laws of nature is nicely summarized in a passage by Ernst Mach with which we want to end our paper:

In our view, laws of nature are a product of our mental need to find our way about in nature [...]. If individual findings later accumulate, there arises a powerful urge to minimize mental effort, to attain economy [...]. The progressive refinement of laws of nature [...] corresponds to a more precise adaption of thought to fact. It is of course not possible to achieve perfect adaptation to every individual and incalculable future fact. It requires abstraction, simplification, schematizing and idealization of the facts, if the laws of nature are to become applicable [...] to actual concrete cases. (Mach 1976: 354-355)

\section{References}

Albert, D. (2000). Time and Chance. Cambridge: Harvard University Press.

Albert, D. (2015). After Physics. Cambridge: Harvard University Press.

Armstrong, D. M. (1983). What is a Law of Nature? Cambridge: Cambridge University Press.

Beebee, H. (2000). The non-governing conception of laws of nature. Philosophy and Phenomenological Research, 61(3), 571-594.

Bird, A. (2007). Nature's Metaphysics: Laws and Properties. Oxford: Oxford University Press.

Callender, C. (2017). What Makes Time Special? Oxford: Oxford University Press. Caton, J. (2014). Resource Bounded Agents. The Internet Encyclopedia of Philosophy, ISSN 2161-0002, http://www.iep.utm.edu/re-bo-ag/.

\footnotetext{
${ }^{23}$ More recently, Callender and Cohen (2010) argue that each science employs its own standards, based on the goals of systematizing their particular domain. Callender (2017) defends a version of the BSA that is similar to our account. According to this version, a set of laws needs to allow for "well-posed Cauchy Problems," which is closely related to what we have above called 'error-tolerance'. Neither of these accounts, however, is proposed as a solution to the challenge for Humeans outlined in $\S 2$.
} 
Callender, C. \& Cohen, J. (2010). Special Sciences, Conspiracy and the Better Best System Account of Lawhood. Erkenntnis, 73(3), 427-447.

Cohen, J. \& Callender, C. (2009). A better best system account of lawhood. Philosophical Studies, 145(1), 1-34.

Deutsch, D. (2012). What is Computation? (How) does nature compute? In. H. Zenil (Ed.), A Computable Universe: Understanding and Exploring Nature as Computation, 551-566. Singapore: World Scientific Publishing.

Dorr, C., \& Hawthorne, J. (2013). Naturalness. In K. Bennett \& D. Zimmerman (Eds.), Oxford Studies in Metaphysics, Volume 8, 3-77. Oxford: Oxford University Press.

Dorst, C. (forthcoming). Toward a Best Predictive System Account of Laws of Nature. British Journal for the Philosophy of Science.

Earman, J. (1984). Laws of nature: the empiricist challenge. In R.J. Bogdan (Ed.), D.M. Armstrong, 191-223. Boston: Reidel.

Earman, J. (1986). A Primer on Determinism. Dordrecht: Reidel.

Elga, A. (2007). Isolation and folk physics. In Price, H., and R. Corry (eds.), Causation, physics and the constitution of reality, 106-119. Oxford: Oxford University Press.

Feynman, R. (1965). The Character of Physical Law. Cambridge, MA: MIT Press.

Frigg, R. (2009). Typicality and the Approach to Equilibrium in Boltzmannian Statistical Mechanics. Philosophy of Science 76: 997-1008.

Frisch, M. (2014) Causal Reasoning in Physics. Cambridge: Cambridge University Press. Hall, N. (2012). Humean reductionism about laws of nature. Unpublished Manuscript. http://philpapers.org/go.pl?id=HALHRA\&proxyId=\&u=http\%3A\%2F\%2Fphilpa pers.org\%2Farchive\%2FHALHRA.pdf

Hall, N. (2015). Humean reductionism about laws of nature. In B. Loewer \& J. Schaffer (Eds.), The Blackwell Companion to David Lewis, 262-277. Oxford: Blackwell. Hawthorne, J. (2006). Quantity in Lewisian Metaphysics. In J. Hawthorne (Ed.), Metaphysical Essays, 229-237. Oxford: Oxford University Press.

Hempel, C. (1965). Aspects of Scientific Explanation and Other Essays in the Philosophy of Science. New York: The Free Press.

Hicks, M. T. (forthcoming). Dynamic Humeanism. British Journal for the Philosophy of Science.

Ismael, J. (2009). “Probability in Deterministic Physics,” The Journal of Philosophy 106: 89-108. 
Ismael, J. (2015). “How to be Humean,” In B. Loewer \& J. Schaffer (Eds.), The Blackwell Companion to David Lewis, 188-205. Oxford: Blackwell.

Jaag, S. (2014). Dispositional essentialism and the grounding of natural modality. Philosophers' Imprint, 14(34): 1-21.

Lange, M. (2009). Laws and Lawmakers: Science, Metaphysics, and the Laws of Nature. Oxford: Oxford University Press.

Lewis, D. K. (1973). Counterfactuals. Oxford: Blackwell.

Lewis, D. K. (1981). Causal Decision Theory. Australasian Journal of Philosophy, 59(1), 5-30.

Lewis, D. K. (1983). New Work for a Theory of Universals. Australasian Journal of Philosophy, 61, 343-377.

Lewis, D. K. (1986a). Philosophical Papers Vol. II. Oxford University Press.

Lewis, D. K. (1986b). On the Plurality of Worlds. Oxford: Basil Blackwell.

Lewis, D. K. (1988). Statements Partly About Observation. Philosophical Papers, 17(1), $1-31$.

Lewis, D. K. (1994). Humean supervenience debugged. Mind, 103(412), 473-490.

Loew, C. (2017). Causation, Physics, and Fit. Synthese, 194(6): 1945-1965.

Loewer, B. (1996). Humean Supervenience. Philosophical Topics, 24(1), 101-127.

Loewer, B. (2007). Laws and Natural Properties. Philosophical Topics, 35(1/2), 313-328.

Mach, E. (1976). Knowledge and Error: Sketches on the Psychology of Enquiry. Dordrecht: Reidel.

Maudlin, T. (2007). The Metaphysics Within Physics. Oxford: Clarendon Press.

Morton, A. (2012). Bounded Thinking: Intellectual Virtues for Limited Agents. Oxford: Oxford University Press.

Roberts, J. T. (2008). The Law-Governed Universe. Oxford: Oxford University Press.

Sober (1975). Simplicity. Oxford: Clarendon Press.

Thagard (1978). The Best Explanation: Criteria for Theory Choice. The Journal of Philosophy, 75, (2), 76-92.

Van Fraassen, B. C. (1989). Laws and Symmetry. Oxford: Oxford University Press.

Wallace, D. (forthcoming). What is Orthodox Quantum Mechanics? Proceedings of the XII International Ontology Congress (held in San Sebastian in 2016).

Weatherson, B. (2015). Humean Supervenience. In B. Loewer \& J. Schaffer (Eds.), The Blackwell Companion to David Lewis, 101-115. Oxford: Blackwell. 
Wilson, J. (2015). Hume's Dictum and Metaphysical Modality: Lewis's Combinatorialism. In B. Loewer \& J. Schaffer (Eds.), The Blackwell Companion to David Lewis, 138158. Oxford: Blackwell.

Woodward, J. (2014). Simplicity in the Best Systems Account of Laws of Nature. British Journal for the Philosophy of Science, 65, 91-123. 